\title{
Risk Factors of Extrapulmonary Tuberculosis in Children
}

\author{
Indah Ratna Sari ${ }^{1}$, Retno Asih Setyoningrum ${ }^{2}$ \\ ${ }^{1}$ Resident, ${ }^{2}$ Lecturer, Department of Child Health, Faculty of Medicine, Universitas Airlangga
}

\begin{abstract}
Background: Tuberculosis (TB) continues to result in high morbidity and mortality in children from resource-limited settings. Extrapulmonary TB had several of clinical appearance and complications. Hence it is important to identify the risk factors for early detection and treatment. The objective of this study is to identify factors associated with extrapulmonary TB in children.

Methods: Observational study conducted at Dr. Soetomo Hospital, Surabaya, Indonesia. Data was collected from medical records of patients who were diagnosed with extrapulmonary TB, aged 1-18 years old in the period of 2010-2018. Data were collected as risk factors were age, nutritional status, BCG (Bacille CalmetteGuerin) immunization status, contact history with adult TB patients, and HIV (human immunodeficiency virus) infection. Each risk factor was analyzed using Chi-square. Risk factors which were statistically significant $(\mathrm{p}<0,05)$ would be analyzed using logistic regression.
\end{abstract}

Results: There were 362 patients diagnosed with extrapulmonary TB. More than a half of them are male $(50,6 \%)$ and $>5$ years old $(52,8 \%)$. Most of them already got BCG immunization (72,9\%) and had normal nutritional status $(73,9 \%)$. Lymphadenitis TB, bone/joint TB and miliary TB were the most extrapulmonary TB in this study. Factors associated with extra pulmonary TB were age (PR 0.51; 95\% CI $=0.38-0.68$; $\mathrm{p}<0.001$ ), nutritional status (PR 3.14; 95\% $\mathrm{CI}=2.29-4.29 ; \mathrm{p}<0.001$ ), and HIV infection (PR 3.66; 95\% $\mathrm{CI}=2.21-6.06 ; \mathrm{p}<0.001)$. In multivariate analysis, age, nutritional status, and HIV infection were statistically significant associated with extrapulmonary $(\operatorname{Exp}(B) 1.921 ; 0.326 ; 0.274$; respectively $p<0.001)$.

Conclusion: Age, nutritional status, and HIV infections are risk factors of extrapulmonary TB in children.

Keywords: Extrapulmonary Tuberculosis, Children, Risk Factors

\section{Introduction}

Tuberculosis (TB) is an infectious bacterial disease caused by Mycobacterium tuberculosis (MTB), which commonly infects the lungs, but can harm any tissue. ${ }^{1}$ World Health Organization (WHO) assumes that there are 133 new TB cases per 100.000 population. ${ }^{2,3}$ TB can spread to extrapulmonary with various percentage

\section{Corresponding author:}

\section{Retno Asih Setyoningrum}

is Doctoral in Department of Child Health, Faculty of Medicine, Universitas Airlangga/ Dr. Soetomo General Hospital. Jl. Mayjen Prof. Dr. Moestopo No. 47, Airlangga, Surabaya, East Java, Indonesia (+6231) 5501681, E-mail: retnosoedijo@yahoo.co.id of incidence according to the organs involved. It can be life threatening and cause morbidity and mortality. ${ }^{4}$ Meningitis TB in one of extrapulmonary TB that can cause death and disability. ${ }^{5}$ The greatest impact of extrapulmonary TB infection in children and immunocompromised individuals is the tendency to develop into severe TB. ${ }^{6}$

WHO providing education to the public about TB transmission and BCG (Bacille Calmette-Guerin) immunization to prevent TB infection. ${ }^{7}$ Although BCG immunization has been used as a TB prevention, but there are still doubts about its effectiveness in preventing TB. ${ }^{8}$ Contact with adult TB patients is considered to be the risk factor for TB in children, ${ }^{9}$ therefore $\mathrm{WHO}$ recommends screening household contact with $\mathrm{TB}$ patients and providing Isoniazid preventive treatment for children. 
Most of the previous studies are about pulmonary TB, meanwhile studies about extrapulmonary TB in children are limited. Therefore, this study aim is to study the risk factors of extrapulmonary TB in children.

\section{Method}

This study was an observational study conducted at Department of Pediatrics Dr. Soetomo General Hospital, Surabaya, Indonesia. The data was collected from medical records of patients who were diagnosed as extrapulmonary TB in January 2010 until December 2018. ${ }^{13}$ Patients aged 1-18 years old, diagnosed with miliary TB, meningitis TB, bone/joint TB, lymphnodes $\mathrm{TB}$, tuberculous pleurisy, cutaneous $\mathrm{TB}$, abdominal TB, reticuloendothelial system $\mathrm{TB}$, renal $\mathrm{TB}$, cardiac $\mathrm{TB}$, and disseminated $\mathrm{TB}$, and received anti tuberculosis drugs in Dr Soetomo General Hospital were included in this study. Some data were collected as risk factors: age, nutritional status (for $\leq 5$ years old using WHO growth chart, ${ }^{11}$ and for $>5$ years old using CDC growth chart $^{12}$ ), BCG immunization status, contact history with adult TB patients, and HIV infection. Each risk factor was analyzed using Chi-square and the one which were statistically significant $(\mathrm{p}<0.05)$ would be analyzed using logistic regression.

\section{Results}

A total 1438 cases of TB were diagnosed during period of January 2010 until December 2018. Among the total 1438 cases, 423 cases were excluded because of incomplete data, 653 cases were pulmonary TB, and 362 cases were extrapulmonary TB. Extrapulmonary TB incidence in male patients were slightly greater than in female $(50.6 \%)$. Most of the patient were $>5$ years old (52.8\%), already got BCG immunization (72.9\%) and had normal nutritional status (73.9\%). The biggest three of extrapulmonary TB in this study were lymphnodes TB (35.9\%), bone/joint TB (22.1\%), and miliary TB (20.2\%) (Table 1). Based on the age group, miliary TB was the most frequent in $<2$ years old group, meanwhile lymph nodes TB was the most frequent in $>2$ years old group (Table 2).

Age was associated with extrapulmonary TB (PR 0.51, CI95\% 0,38-0,68, $\mathrm{p}<0.001$ ), malnutrition and HIV infection children were more likely to have extrapulmonary TB (PR 3.14, CI95\% 2.29-4.29, p $<0.001$ and PR 3.66, CI 95\% 2.21-6.06, $\mathrm{p}<0.001$ respectively) (Table 3). Our subjects were divided into 3 age groups to determine significant risk factor in each group. This study found that malnutrition and HIV infected children were 2-4 times as likely to have significant risk factors of extrapulmonary TB in all age group (Table 4, 5, 6). In multivariate analysis, age, nutritional status, and HIV infection were statistically significant associated with extrapulmonary $(\operatorname{Exp}(B) 1.921 ; 0.326 ; 0.274$; respectively $\mathrm{p}<0.001$ ) (Table 7 ).

Table 1. Characteristic of Subjects

\begin{tabular}{|l|l|l|}
\hline & Extrapulmonary TB (N=362) n (\%) & $\begin{array}{l}\text { Pulmonary TB (N=653) } \\
\mathbf{n}(\%)\end{array}$ \\
\hline Gender & & \\
\hline Male & $183(50.6)$ & $330(50,5)$ \\
\hline Female & $179(49.4)$ & $323(49,5)$ \\
\hline Nutritional Status & & \\
\hline Normal & $242(66.9)$ & $564(86,4)$ \\
\hline Malnutrition & $120(33.1)$ & $89(13,7)$ \\
\hline Age & & \\
\hline$<2$ year old & $78(21.5)$ & $230(35,2)$ \\
\hline $2-5$ year old & $93(25.7)$ & $140(21,4)$ \\
\hline
\end{tabular}




\section{Cont... Table 1. Characteristic of Subjects}

\begin{tabular}{|c|c|c|}
\hline$>5$ year old & $191(52.8)$ & $281(43,0)$ \\
\hline \multicolumn{3}{|l|}{ TB contact } \\
\hline Yes & 179 (49.4) & $392(53,7)$ \\
\hline No & $183(50.6)$ & $338(46,3)$ \\
\hline \multicolumn{3}{|c|}{ BCG Immunization } \\
\hline Yes & $246(72.9)$ & $472(72,3)$ \\
\hline No & $98(27.1)$ & $181(27,7)$ \\
\hline \multicolumn{3}{|l|}{ HIV Infection } \\
\hline Yes & $19(5.2)$ & $110(16,8)$ \\
\hline No & $343(94.8)$ & $543(83,2)$ \\
\hline
\end{tabular}

Table 2. Extra Pulmonary Tuberculosis Incidence based on Age

\begin{tabular}{|c|c|c|c|c|}
\hline & & Age & & \\
\hline & n (\%) & $\begin{array}{l}<2 \text { year old }(n=78) \\
\text { n }(\%)\end{array}$ & $\begin{array}{l}2-5 \text { year old } \\
(n=93) \\
n(\%)\end{array}$ & $\begin{array}{l}>5 \text { year old } \\
(n=191) \\
n(\%)\end{array}$ \\
\hline Meningitis TB & $27(2,7)$ & $15(19.2)$ & $9(9.7)$ & $3(1.6)$ \\
\hline Bone/ Joint TB & $80(7,9)$ & $7(9)$ & $24(25.8)$ & 49 (25.7) \\
\hline Lymph Nodes TB & $130(12,8)$ & $22(28.2)$ & $39(41.9)$ & $69(36.1)$ \\
\hline Tuberculous pleurisy & $6(0,6)$ & 0 & $2(2.2)$ & $4(2.1)$ \\
\hline Cutaneous TB & $7(0,8)$ & $1(1.3)$ & 0 & $6(3.1)$ \\
\hline Abdominal TB & $22(2,2)$ & 0 & $5(5.4)$ & $17(8.9)$ \\
\hline Reticuloendothelial TB & $1(0,1)$ & 0 & $1(1.1)$ & 0 \\
\hline Renal TB & $3(0,3)$ & 0 & 0 & 0 \\
\hline Cardiac TB & $73(7,2)$ & 0 & 0 & $3(1.6)$ \\
\hline Miliary TB & $13(1,3)$ & $27(34.6)$ & $11(11.8)$ & $35(18.3)$ \\
\hline Disseminated TB & $27(2,7)$ & $6(7.7)$ & $2(2.2)$ & $5(2.6)$ \\
\hline
\end{tabular}


Tabel 3. Risk Factors of Extrapulmonary TB

\begin{tabular}{|c|c|c|c|c|}
\hline Risk Factors & $\begin{array}{l}\text { Case } \\
\mathrm{n}(\%)\end{array}$ & PR & CI 95\% & $\mathrm{P}$ value \\
\hline $\begin{array}{l}\text { Age } \\
<2 \text { year old } \\
>2 \text { year old }\end{array}$ & $\begin{array}{l}78(21,5) \\
284(82,1)\end{array}$ & 0.51 & $0,38-0,68$ & $<0,001^{*} \#$ \\
\hline $\begin{array}{l}\text { Nutritional status } \\
\text { Malnutrition } \\
\text { Normal }\end{array}$ & $\begin{array}{l}120(33,1) \\
242(66,9)\end{array}$ & 3.14 & $2,29-4,29$ & $<0,001^{*} \#$ \\
\hline $\begin{array}{l}\text { BCG immunization } \\
\text { Yes } \\
\text { No }\end{array}$ & $\begin{array}{l}264(72,9) \\
98(27,1)\end{array}$ & 0.97 & $0,73-1,29$ & $0,883 \#$ \\
\hline $\begin{array}{l}\text { Contact with TB patients } \\
\text { Yes } \\
\text { No }\end{array}$ & $\begin{array}{l}179(49,4) \\
183(50,6)\end{array}$ & 1.14 & $0,88-1,47$ & $0,326 \#$ \\
\hline $\begin{array}{l}\text { HIV infection } \\
\text { Yes } \\
\text { No }\end{array}$ & $\begin{array}{l}19(5,2) \\
343(94,8)\end{array}$ & 3.66 & $2,21-6,06$ & $<0,001^{*} \#$ \\
\hline
\end{tabular}

${ }^{*} \mathrm{p}<0.05$ was considered statistically significant

${ }^{\#}$ Chi Square test was used

$(\mathrm{PR}=$ Prevalent Ratio $; \mathrm{CI}=$ Confidence Interval $)$

Table 4. Risk Factors of Extrapulmonary TB in age group

\begin{tabular}{|c|c|c|c|c|c|c|c|c|c|c|c|c|}
\hline \multirow{2}{*}{ Risk Factor } & \multicolumn{4}{|c|}{$<2$ years old group $(n=78)$} & \multicolumn{4}{|c|}{ 2-5 years old group $(n=93)$} & \multicolumn{4}{|c|}{$>5$ years old group $(n=191)$} \\
\hline & $\begin{array}{l}\text { Case } \\
\text { n (\%) }\end{array}$ & PR & CI 95\% & P value & $\begin{array}{l}\text { Case } \\
\text { n (\%) }\end{array}$ & PR & CI $95 \%$ & P value & \begin{tabular}{|l} 
Case \\
n (\%)
\end{tabular} & PR & $\begin{array}{l}\text { CI } \\
95 \%\end{array}$ & P value \\
\hline $\begin{array}{l}\text { Nutritional status } \\
\text { Malnutrition } \\
\text { Normal }\end{array}$ & $\begin{array}{l}25(32,1) \\
53(67,9)\end{array}$ & 3,55 & $1,90-6,61$ & $<0,001 * \#$ & $\begin{array}{l}24(25,8) \\
69(74,2)\end{array}$ & 2,89 & $1,43-5,89$ & $0,004 * \#$ & $\begin{array}{l}71(37,2) \\
120(62,8)\end{array}$ & 2,95 & $\begin{array}{l}1,92- \\
4,53\end{array}$ & $<0,001^{* \#}$ \\
\hline $\begin{array}{l}\text { BCG immunization } \\
\text { Yes } \\
\text { No }\end{array}$ & $\begin{array}{l}59(75,6) \\
19(24,4)\end{array}$ & 1,00 & $0,55-1,82$ & $1,000 \#$ & $\begin{array}{l}74(79,6) \\
19(20,4)\end{array}$ & 0,69 & $0,37-1,29$ & $0,278 \#$ & $\begin{array}{l}131(68,6) \\
60(31,4)\end{array}$ & 1,04 & $\begin{array}{l}0,69- \\
1,55\end{array}$ & $0,919 \#$ \\
\hline $\begin{array}{l}\text { Contact with TB } \\
\text { patients } \\
\text { Yes } \\
\text { No }\end{array}$ & $\begin{array}{l}40(51,3) \\
38(48,7)\end{array}$ & 1,04 & $0,62-1,73$ & $0,897 \#$ & $\begin{array}{l}42(45,2) \\
51(54,8)\end{array}$ & 1,53 & $0,90-2,59$ & $0,141 \#$ & $\begin{array}{l}94(49,2) \\
97(50,8)\end{array}$ & 1,05 & $\begin{array}{l}0,73- \\
1,51\end{array}$ & $0,851 \#$ \\
\hline $\begin{array}{l}\text { HIV infected } \\
\text { Yes } \\
\text { No }\end{array}$ & $\begin{array}{l}4(5,1) \\
74(94,9)\end{array}$ & 3,78 & $\begin{array}{l}1,30- \\
10,94\end{array}$ & $0,008^{* \#}$ & $\begin{array}{l}6(6,5) \\
87(93,5)\end{array}$ & 4,29 & $\begin{array}{l}1,72- \\
10,74\end{array}$ & $0,001 * \#$ & \begin{tabular}{|l}
$9(4,7)$ \\
$182(95,3)$
\end{tabular} & 3,16 & $\begin{array}{l}1,49- \\
6,71\end{array}$ & $0,002 * \#$ \\
\hline
\end{tabular}

$*_{\mathrm{p}}<0.05$ was considered statistically significant 
${ }^{\#}$ Chi Square test was used

$(\mathrm{PR}=$ Prevalent Ratio $\mathrm{CI}=$ Confidence Interval $)$

Table 5. Multivariate Analysis of Extrapulmonary TB Risk Factors in Children

\begin{tabular}{|l|l|l|l|}
\hline Risk Factors & $\operatorname{Exp(B)}$ & $\begin{array}{l}\text { CI } \\
\mathbf{9 5 \%}\end{array}$ & P value \\
\hline Age & 1,921 & $\begin{array}{l}1,41- \\
2,62\end{array}$ & $<0,001^{* \#}$ \\
\hline Nutritional status & 0,326 & $\begin{array}{l}0,24- \\
0,45\end{array}$ & $<0,001^{* \#}$ \\
\hline HIV infected & 0,274 & $\begin{array}{l}0,16- \\
0,46\end{array}$ & $<0,001^{* \#}$ \\
\hline Constant & 0,754 & & \\
\hline
\end{tabular}

${ }^{*} \mathrm{p}<0.05$ was considered statistically significant

${ }^{\#}$ Logistic Regression test was used

$(\mathrm{CI}=$ Confidence Interval $)$

Abbreviations and Symbols

AIDS

: Acquired Immune Deficiency

Syndrome

$\begin{array}{ll}\text { ART } & : \text { Antiretroviral Therapy } \\ \text { BCG } & : \text { Bacille Calmette-Guerin } \\ \text { BMI } & : \text { Body Mass Index } \\ \text { CD } & : \text { Cluster of Differentiation } \\ \text { CDC } & : \text { Centers for Disease Control }\end{array}$

and prevention

CI

HIV Virus

$\begin{array}{ll}\text { INH } & : \text { Isoniazid } \\ \text { MTB } & : \text { Mycobacterium Tuberculosis } \\ \text { PR } & : \text { Prevalent Ratio } \\ \text { OR } & : \text { Odd Ratio } \\ \text { TB } & : \text { Tuberculosis }\end{array}$

WHO : World Health Organization

\section{Discussion}

In our study, there were 362 extrapulmonary TB patients. Most of our subjects (52.8\%) were over 5 years old, and male patients were slightly more frequent (50.6\% vs $49.4 \%)$. Saraswati et al mentioned that there was association between TB infection and gender, it might be affected by the sexual hormones and the children's lifestyle, ${ }^{15}$ immunity response to vaccine, and immunoglobin related to gender. ${ }^{16}$ Among the 362 extrapulmonary TB patients, $2.7 \%$ were disseminated TB, $2.7 \%$ were meningitis $\mathrm{TB}, 1.3 \%$ were miliary $\mathrm{TB}$, and $72.9 \%$ were already got $\mathrm{BCG}$ immunization. Although, BCG immunization can provide protection against disseminated TB infection, but it could not give maximum protection against the other types of TB. ${ }^{15}$

In the age group of $<2$ years old, the three highest prevalence were miliary TB (34.6\%), lymphnodes TB (28.2\%), and meningitis TB (19.2\%). Wallgren in 1948 stated that hematogenous spread occured 1-3 months after primary infection would develop into TB meningitis and miliary TB in young children, ${ }^{17}$ and WHO estimated that more than $50 \%$ of TB cases in children younger than 5 years old were disseminated TB. ${ }^{2}$ Meanwhile in age group of 2-5 years old and more than 5 years old, lymphnodes TB had the highest prevalence (41.9\% and $36.1 \%$, respectively). Previous study found similar results. ${ }^{18}$

In this study, age was associated with extrapulmonary TB (PR 0.51, CI 95\% 0.38-0.68, $<<0.001$ ). Marais et al mentioned that children aged less than 1 year old had higher prevalence of disseminated TB compared with children aged 1-2 years old and more than 5 years old, ${ }^{19}$ because infants' immunity system were different from adults and it could not function properly. Therefore, infants have 5-10 times greater risk of suffering active and severe TB. ${ }^{20}$

This study found that malnutrition was a significant risk factor for extrapulmonary TB in all ages group (PR 2.89-3.55 $\mathrm{p}<0.05)$. Other studies has similar result. ${ }^{3}$ Immune mechanism against MTB infection is depend on interaction of monocytes, macrophages, T lymphocytes and cytokines in which inadequate nutrition will reduce its interaction. ${ }^{21}$ Malnutrition children have less type 1 
cytokines (Interleukin-2 and Interferon- $\gamma$ ) which were primary immunity mediators. ${ }^{22}$ Changes of these cellmediated immune could increase the susceptibility to infection. There are many studies about malnutrition and $\mathrm{TB}$, but it is difficult to prove whether malnutrition or TB that precede one another. Weight loss and malnutrition in TB patients can be caused by decreased food intake or factors due to TB disease itself. Altered metabolism of TB can lead to "anabolic blocks," where food protein is used more for energy production rather than anabolism. ${ }^{23}$

This study showed that BCG immunization status was not associated with extrapulmonary TB. Previous studies found similar result. ${ }^{24}$ There are many factors affecting immune response to BCG vaccine, such as where the immunization is done, maternal immunity factors, late $\mathrm{BCG}$ vaccine, and others. ${ }^{25}$ The cell wall component of BCG vaccine will occupy macrophage receptors as is done by $\mathrm{MTB}$, therefore BCG immunization has no protective effect if it is given to people who have been already infected or sensitized by MTB. Contact with adult TB patient was not associated with extrapulmonary TB in this study. Many factors affect host defense against pathogens including how MTB induce necrosis by destructing mitochondria inner membranes and preventing plasma membrane repairments which is mediated by lysosomal and Golgi. If MTB cannot be killed by innate immunity response, therefore MTB will replicate and spread. ${ }^{26}$

Among the 362 extrapulmonary TB patients, 5\% of our subjects had HIV infection and children with HIV infection were 3-4 times as likely to have extrapulmonary TB. Previous studies stated that HIV infection is associated with TB infection on children. ${ }^{24} \mathrm{HIV}$ infects T CD4+ and macrophage and MTB also mainly infect macrophage in which to eradicate intracellular microbial pathogens. Therefore, low T CD4+ cells will increase risk of TB infection. TB and HIV are chronic and progressive disease which will impair host protection by promoting an immunoregulatory phenotype characterized by an attenuated $\mathrm{T}$ cell response and leads to failure of normal homeostatic control of the inflammatory response. ${ }^{27}$

\section{Conclusion}

Age, nutritional status, and HIV infections are risk factors of extrapulmonary TB. Meanwhile BCG immunization and contact with adult TB patients are not proven as risk factors.

Ethical Clearance : Approved by Ethical committee in health research Dr. Soetomo General Hospital Surabaya, 0943/KEPK/II/2019

\section{Source of Funding : self}

Conflict of Interest : there are no conflict of interest

\section{References}

1. Bloom B, Atun R, Cohen T, Dye C, Fraser H, Gomez G, et al. Tuberculosis. In: Major Infectious Diseases. 2017. p. 233-313.

2. WHO. Roadmap towards ending TB in children and adolescents [Internet]. Who. 2018. Available from: https://www.who.int/tb/publications/2018/ tb-childhoodroadmap/en/

3. WHO. Global Tuberculosis Report 2017: Leave no one behind - Unite to end TB [Internet]. WHO Technical Report Series;727.2017. 146 p. Available from: http://www.who.int/tb/publications/global_ report/gtbr2017_main_text.pdf?ua=1

4. Bukhary ZA, Alrajhi AA. Extrapulmonary tuberculosis, clinical presentation and outcome. Saudi Med J. 2004;25(7):881-5.

5. Mezochow A, Thakur K, Vinnard C. Tuberculous Meningitis in Children and Adults: New Insights for an Ancient Foe. Curr Neurol Neurosci Rep. 2017;17(11):85.

6. Triasih R, Helmi R, Laksanawati I. Clinical profile and outcome of extrapulmonary tuberculosis in children in Indonesia. Pediatr Respirol Crit Care Med. 2018;2(3):51.

7. WHO. SAGE Working Group on BCG vaccines (October 2016 to February 2018). 2016.

8. Kumar R, Singh BK, Iqbali T. Comparison of Tuberculous meningitis in children with or without BCG Scar. Int J Med Paediatr Oncol. 2016;2(4):142-5.

9. Donald PR, Marais BJ, Barry CE. Age and the epidemiology and pathogenesis of tuberculosis. Lancet. 2010;375(9729):1852-4.

10. Ministry of Health of the Republic of Indonesia. Petunjuk Teknis Manajemen dan Tatalaksana TB Anak. 2016. 9-28 p.

11. WHO. Child growth standards [Internet]. Available from: https://www.who.int/childgrowth/standards/ 
chart_catalogue/en/

12. Kuczmarski R, Ogden C, Guo S, Al E. 2000 CDC growth charts for the United States: Methods and development. Vol. 11, National Center for Health Statistics. Vital Health Stat. 2002. 27-32 p.

13. Alavi SM, Salmanzadeh S, Bakhtiyariniya P, Albagi A, Hemmatnia F, Alavi L. Prevalence and treatment outcome of pulmonary and extrapulmonary pediatric tuberculosis in southwestern Iran. Casp J Intern Med. 2015;6(4):213-9.

14. García-Rodríguez JF, Álvarez-Díaz H, LorenzoGarcía MV, Mariño-Callejo A, Fernández-Rial Á, Sesma-Sánchez P. Extrapulmonary tuberculosis: Epidemiology and risk factors. Enferm Infecc Microbiol Clin. 2011;29(7):502-9.

15. Saraswati LD, Ginandjar P, Widjanarko B, Puspitasari RA. Epidemiology ofChild Tuberculosis (A Cross-Sectional Study at Pulmonary Health Center Semarang City, Indonesia). IOP Conf Ser Earth Environ Sci. 2018;116(1):012081.

16. Klein SL, Flanagan KL. Sex differences in immune responses. Nat Rev Immunol. 2016;16(10):626-38.

17. Perez-Velez CM, Roya-Pabon CL, Marais BJ. A systematic approach to diagnosing intra-thoracic tuberculosis in children. J Infect [Internet]. 2017;74:S74-83.

18. Santiago-García B, Blázquez-Gamero D, BaqueroArtigao F, Ruíz-Contreras J, Bellón JM, MuñozFernández MA, et al. Pediatric extrapulmonary tuberculosis: Clinical spectrum, risk factors and diagnostic challenges in a low prevalence region. Pediatr Infect Dis J. 2016;35(11):1175-81.
19. Marais BJ, Hesseling AC, Gie RP, Schaaf HS, Beyers N. The burden of childhood tuberculosis and the accuracy of community-based surveillance data. Int J Tuberc Lung Dis. 2006;10(3):259-63.

20. Driessche K Vanden, Persson A, Marais BJ, Fink PJ, Urdahl KB. Immune Vulnerability of Infants to Tuberculosis. Clin Dev Immunol. 2013;2013:781320.

21. Rook G, Hernandez-Pando R. The pathogenesis of tuberculosis. Annu Rev Microbiol. 1996;50:25984.

22. Rodríguez L, González C, Flores L, JiménezZamudio L, Graniel J, Ortiz R. Assessment by flow cytometry of cytokine production in malnourished children. Clin Diagn Lab Immunol. 2005;12(4):502-7.

23. Sinha P, Davis J, Wanke C, Salgame P, Mesick $\mathrm{J}$, Horsburgh Jr C, et al. Undernutrition and Tuberculosis: Public Health Implications. J Infect Dis. 2019;219(9):1356-63.

24. Jain SK, Ordonez A, Kinikar A, Gupte N, Thakar $\mathrm{M}$, Mave V, et al. Pediatric tuberculosis in young children in India: A prospective study. Biomed Res Int. 2013;2013:783698.

25. Dockrell HM, Smith SG. What have we learnt about BCG vaccination in the last 20 years? Front Immunol. 2017;8:1134.

26. Lerner TR, Borel S, Gutierrez MG. The innate immune response in human tuberculosis. Cell Microbiol. 2015;17(9):1277-85.

27. Bell LCK, Noursadeghi M. Pathogenesis of HIV-1 and mycobacterium tuberculosis co-infection. Nat Rev Microbiol [Internet]. 2018;16(2):80-90. 